\title{
CUBO DE RUBIK: UMA FERRAMENTA PARA O ENSINO DE FÍSICA.
}

\author{
Geovani R. Da Silva*, Petrus H. R. dos Anjos* \\ * Departamento de Física-Campus Catalão \\ Universidade Federal de Goiás \\ Catalão GO, Brazil
}

Emails: geovanirdasilva@gmail.com, petrus@ufg.br

\begin{abstract}
The famous Rubik's cube offers interesting opportunities to teach modern physical concepts and to develop problem-solving skills. This article briefly discuss some ideas for a physics teacher to use this tool.
\end{abstract}

Keywords- Rubik's cube, Teaching physics, Problem-solving skills

Resumo- O famoso cubo de Rubik oferece oportunidades interessantes para ensinar conceitos físicos modernos e para desenvolver habilidades de resolução de problemas. Este artigo discute brevemente algumas ideias para um professor de física usar esta ferramenta.

Palavras-chave - Cubo de Rubik, Ensino de física e Habilidade de resolução de problemas

\section{Introdução}

Assim como na vida, a habilidade na solução de problemas desempenha um papel importante no ensino-aprendizagem da física. Uma grande habilidade na resolução de problemas, usualmente denota um alto desenvolvimento de competências reguladoras (planejamento, monitoramento, avaliação) e transformadoras (questionamento, formulação de hipóteses, investigação e interpretação) importantes para o aprendizado (Ref. [1]). Infelizmente, muitas vezes o ensino tradicional leva os estudantes a resolver problemas de física de maneira puramente mecânica (Ref. [2]): o problema proposto no livro solicita uma determinada quantia e fornece estritamente os dados necessários, então "letras vem a memória", as formulas onde aparecem essas letras são utilizadas, se insere os números na fórmula, e - problema resolvido! Não há espaço para se pensar sobre o conteúdo e não se desenvolve nenhuma das habilidades acima. Problemas deste tipo, que não demandam raciocínio, comuns em livros-texto de física, são bastante distantes dos problemas que ocorrem na vida real. Resolver problemas, especialmente de física, exigem a aplicação de uma teoria/conhecimento previamente dominado (Ref. [3]), capacidade de compreensão do problema, capacidade de planejamento, de execução e de análise (Ref.[4]). Possivelmente esta é uma das motivações por trás da célebre frase de Einstein "Education is what remains after one has forgotten everything he learned in school". Na tentativa de alterar este panorama, diversas estratégias têm sido empregadas, entre elas o uso de atividades manipulativas, jogos e desafios (Ref. [5]). Neste trabalho, propomos continuar a explorar este tipo de estratégia utilizando o quebra-cabeça conhecido como cubo mágico. Também chamado de cubo de Rubik, em homenagem a seu inventor o húngaro Erno Rubik. Rubik fabricou o primeiro protótipo do cubo em
1974, para ajudar a ilustrar o conceito da terceira dimensão para alunos de arquitetura. O cubo começou a ser comercializado em 1980, e em janeiro de 2009, 350 milhões de cubos já haviam sido vendidos em todo o mundo, tornando-o jogo mais vendido. Ele é considerado um quebra-cabeça bastante difícil, que desafia o raciocínio espacial, a memória e a capacidade de planejamento. Por exemplo, no filme "Pursuit of Happyness", que é baseado na história real de Chris Gardner; o personagem principal consegue um emprego ( e termina por ganhar milhões) após impressionar seu futuro empregador resolvendo o cubo de Rubik.

A dificuldade e frustração com as seguidas falhas nas tentativas iniciais de se resolver o cubo são bastante semelhantes às enfrentadas pelos estudantes ao se depararem com problemas mais complexos de ciências e matemática. Mas como no caso do cubo de Rubik, a dificuldade da tarefa e o fato de conseguir resolve-la, após muito trabalho, faz toda a frustração valer a pena: sentese orgulho da realização e satisfação por aprender algo novo. Sente-se que se podemos aprender uma tarefa difícil como o cubo, também podemos aprender outras disciplinas complexas.

Neste trabalho discutiremos que o aumento na autoconfiança dos estudantes não é o único benefício do cubo no ensino. Do ponto de vista de resolução de problemas, um grande apelo do cubo de Rubik é seu resultado final. É perfeitamente claro, quando ele está resolvido ou não. Não há nenhuma ambiguidade na resposta, a única maneira de melhora-la é atingi-la mais rapidamente. Para fazê-lo, tal como ocorre na prática da ciência, se requer aprendizado de novas técnicas e operações que serão de aprendizado difícil no começo, mas que com prática e dedicação se tornam naturais.

Do ponto de vista do ensino de física, o apelo do cubo de Rubik é a possibilidade de utiliza-lo para introduzir e ilustrar conceitos modernos de 
física, oferecendo um modelo educacional para explorar um mundo desconhecido de maneira científica. As regras de movimento do cubo (impostas por suas simetrias) fixam leis de conservação, que reduzem o número de estados permitidos, mas que fazem com que seja difícil chegar a um estado desejado (Ref. [?]). A impossibilidade de se atingir qualquer padrão imaginado abre campo para a discussão da ergodicidade do problema. O grande número de estados sugere uma abordagem estatística para expressar a irreversibilidade experimentada por quem manipula o cubo (Ref. [7]). E mesmos as simetrias do Eightfold Way presente no modelo de quarks pode ser ilustrada com o uso do cubo de Rubik (Ref. [8]). Tais características tornam o cubo de Rubik uma ferramenta educacional promissora para professores de Física em diversos níveis de ensino.

O restante deste trabalho está organizado da seguinte forma: Na seção 2, discutiremos brevemente o funcionamento do cubo de Rubik. Na seção 3, apresentaremos uma estratégia de solução para o Cubo e apresentamos uma possível atividade para estudantes. Na seção 4, apresentamos um pequeno roteiro para ensinar em sala de aula como resolver o cubo utilizando a estratégia apresentada na seção 3. Na seção 5, discutiremos alguns dos benefícios do cubo de Rubik no ensino. Na seção 6, esboçaremos algumas conclusões preliminares.

\section{O Cubo de Rubik e sua mecânica}

Cubo de Rubik é um quebra-cabeça tridimensional, cujo objetivo é o de fazer com que cada lado do cubo tenha apenas uma cor, como mostrado na Figura 1.

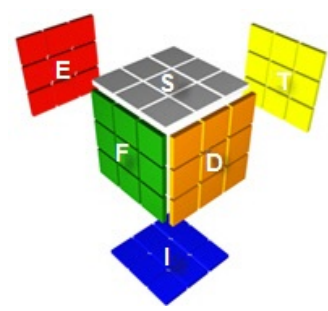

Figura 1: Diagrama de um cubo de Rubik resolvido. Os seis lados são: superior $(\mathrm{S})$, frontal $(\mathrm{F})$, e direito (D) os lados são visíveis. Os restantes lados esquerdo (E), traseiro (T) e inferior (D) que são mostrados pelas imagens projetadas.

Cada uma das três divisões horizontais do cubo é chamada de linha, ao passo que se denomina coluna cada uma das três divisões verticais do cubo. O cubo é construído de tal maneira que linhas e colunas podem girar para ambos os lados (ver Figura 2). Assim a cada instante pode se realizar 12 movimentos (de $1 / 4$ de volta) com o cubo, mudando sua configuração. Dessa forma mesmo após alguns poucos movimentos, as cores do cubo terminam embaralhadas. O desafio do quebra-cabeça é retornar as peças para suas posições originais.

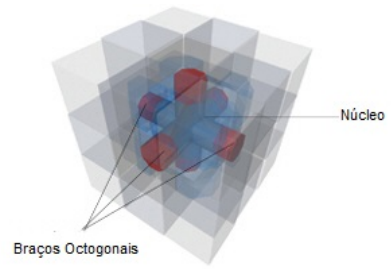

Figura 2: Diagrama do núcleo de um cubo de Rubik. O núcleo permite que cada linha e coluna do cubo girem para ambos os lados.

É difícil entender a mecânica do cubo sem primeiro conhecer os seus componentes. O cubo de Rubik consiste em duas componentes distintas: o núcleo e as peças (cubos) exteriores. A forma do núcleo consiste em um cubo central imaginário com seis braços octogonais ligados a cada uma das faces (Figura. 2). Cada octógono é anexado ao cubo central permitindo a rotação livre em qualquer direção. As faces octogonais de cada um destes braços são perpendiculares ao cubo imaginário. As peças (cubos) exteriores são anexadas ao núcleo e podem ser divididas em três tipos: as centrais, as laterais e vértices (Figura 3). Há um total de seis peças centrais (Tabela 1), cada um tem apenas uma face visível na construção final do cubo. Cada peça central é ligada perpendicularmente a um dos braços octogonais, as peças centrais nunca se movem em relação ao núcleo. Há doze peças laterais que formam as arestas do cubo, cada uma destas peças possui duas faces visíveis. Estas peças são conectadas ao núcleo por conectores cúbicos localizados nas faces não visíveis. Finalmente, há oito peças de vértice, cada uma com três faces visíveis. Cada peça de vértice possui um conector localizado na aresta oposta a de suas faces visíveis. Os conectores das peças laterais e de vértice se encaixam nas arestas dos braços octogonais.
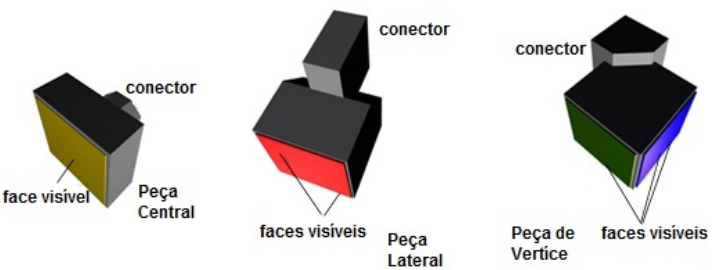

Figura 3: Componentes do Cubo de Rubik

Juntas, estas vinte e seis peças compõem a parte visível do cubo de Rubik. Cada uma delas é presa firmemente por seus conectores aos braços do núcleo, impedindo que as peças adjacentes saltem para fora. Como cada um dos braços oc- 
Tabela 1: Peças do Cubo de Rubik.

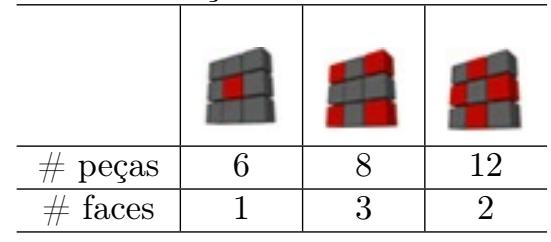

togonais pode rodar, as camadas do cubo (linhas e colunas) podem ser rotacionadas a partir da sua posição original. Quando um dos braços octogonais do núcleo gira, giram também os nove cubos externos conectados com ele, uma vez que estes cubos estão conectados aos núcleos e travados por seus vizinhos. Com rotações de múltiplos de $\frac{\pi}{4}$, o cubo pode ser devolvido à sua forma original, mas é pouco provável que se retorne a sua posição original . Depois de apenas algumas rotações aleatórias de diferentes lados, as cores em cada uma das faces estarão muito bem embaralhadas.

\section{Resolvendo o cubo}

Atualmente existe uma infinidade de métodos de solução para o cubo de Rubik, muitos dos quais disponíveis na web (veja por exemplo : www.ryanheise.com/cube/beginner.html). Tais métodos utilizam diferentes sequências de movimentos ou dividem o problema de resolver o cubo em diferentes problemas menores que uma vez resolvidos conduzem a solução final.

Nesta seção será apresentado um método simples, que utiliza apenas 3 sequências de movimento para mudar ou ajustar peças diferentes. $\mathrm{Na}$ terminologia empregada nesta seção ajustar uma peça significa a alterar a direção das cores de uma peça (Figura 4). Comutar duas peças refere-se a trocar a posição de duas peças diferentes (Figura 5).

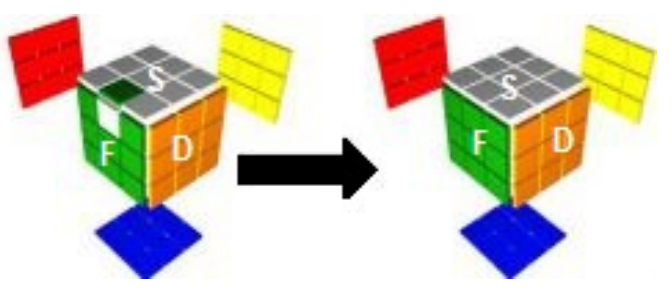

Figura 4: Ajustar uma peça

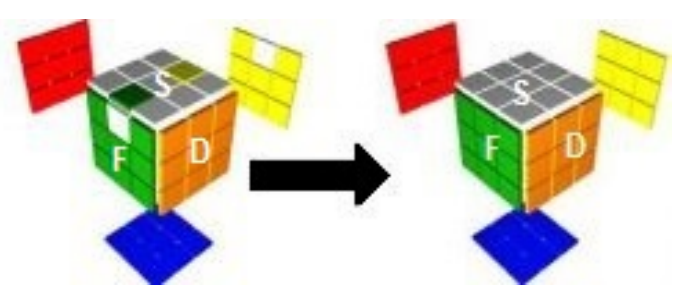

Figura 5: Comutar duas peças.
Sequência 1 -Um movimento para ajustar dois vértices consecutivos (nada altera mais é alterado) (Figura 6): $i$. Segurar o cubo de modo que os dois vértices a serem ajustados estejam na face superior; ii. Gire a coluna central para cima; iii. Gire a linha superior para a esquerda; $i v$. Repita as etapas 2 e 3 mais duas vezes; $v$. Gire a linha superior para esquerda; vi. Gire a coluna central para baixo; vii. Repita os passos 5 e 6 mais duas vezes; viii. Rodar a linha superior para a esquerda duas vezes.

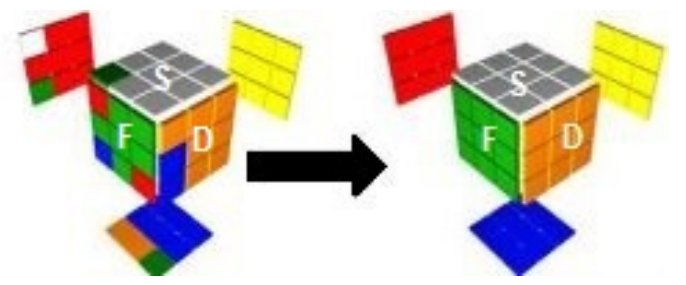

Figura 6: Ajustar vértices consecutivos.

Sequência 2 - Um movimento para ajustar três vértices de uma face (Altera arestas adjacentes) (Figura 7): $i$. Segure o cubo de forma que as arestas a serem alteradas estejam na face superior e voltadas para você e o canto inalterado fique na parte superior direita; ii. Gire a linha superior para a direita; iii. Gire a face frontal no sentido horário duas vezes; $i v$. Gire a linha superior para esquerda; $v$. Gire a face frontal no sentido horário; vi. Gire a linha superior para a direita; vii. Gire a face frontal no sentido horário; viii. Gire a linha superior para a esquerda; $i x$. Gire a face superior no sentido horário duas vezes.

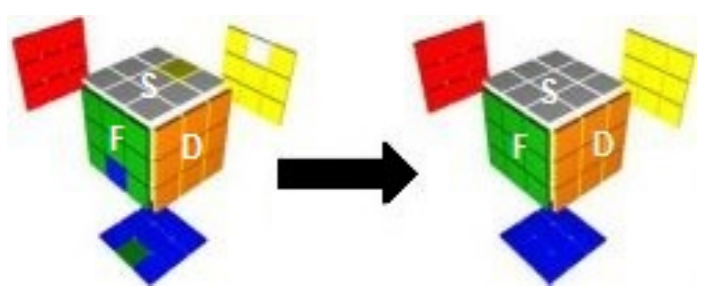

Figura 7: Ajustar três cantos

Sequência 3 - um movimento para mudar as posições de três peças laterais em um mesmo plano (altera nada mais) (Figura 8): $i$. Segurar o cubo de modo que o plano a ser alterado é o seja uma coluna central; ii. Posicione a quarta peça lateral no plano inferior e na face oposta a que esta próxima de você; iii. Gire a coluna central; $i v$. Gire a linha superior para a esquerda duas vezes; $v$. Gire a coluna central para baixo; vi. Gire a linha superior para a esquerda duas vezes.

Estas três sequências de movimento são vantajosas, pois seus efeitos são facilmente compreendidos e são de simples memorização. O planejamento de um algoritmo para resolver o Cubo de Rubik utilizando estes três seria um projeto de 


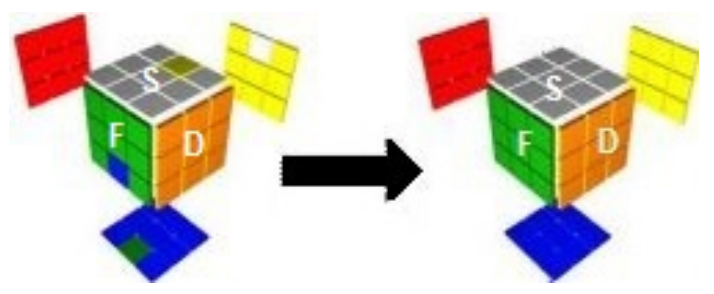

Figura 8: Mudar a posição das arestas na mesma face.

ciências bastante interessante e factível para alunos que demonstrem gosto por resolver quebracabeças, boa capacidade de raciocínio espacial e/ou já conheçam como resolver o cubo de $\mathrm{Ru}-$ bik. O objetivo do aluno neste trabalho seria determinar se as três sequências de movimentos são suficientes para resolver o cubo. Em outras palavras, se é possível resolver o cubo com apenas estes três sequências de movimento, ou que se seriam necessários movimento adicionais.

Uma estratégia para resolver o cubo de Rubik utilizando estes 3 movimentos poderia ser dividida em 5 passos:

Passo 1: Fazer uma cruz em um lado do cubo. Fazer a cruz é simplesmente uma questão de inserção uma a uma cada uma das 4 peças laterais, um a um, em torno da peça central sem remover as peças que já foram colocadas. Gire a cruz até algumas das cores das peças laterais que formam a cruz combinem com as peças nos lados do cubo. Se apenas uma cor combinar, continue rotacionando a cruz: É sempre possível obter a combinação de duas cores.

No caso de se obter apenas duas peças laterais combinando, as peças laterais que não combinarem devem ser trocadas de posição. Existem duas possibilidades diferentes. Ou as duas peças laterais a serem trocadas estão próximas umas das outras, ou estão em lados opostos do cubo. Ambos os casos podem ser tratados pela mesma estratégia. Em primeiro lugar, desloque um destas peças para a face oposta do cubo. Dessa forma se pode mover a camada oposta de forma independente da camada onde foi feita a cruz. A seguir, gire a peça trocada sobre a camada superior até que ela fique posicionada diretamente acima da sua posição correta. Finalmente, gire esta peça novamente para a camada onde está a cruz. Este mesmo movimento também moverá o outra peça trocado para a camada oposta, onde ela pode ser levada para a posição correta utilizando a mesma estratégia no sentido inverso. Ao fim deste procedimento o cubo deve se encontrar num padrão análogo ao da Figura 9. Este é um passo muito importante, visto que ele deverá ser repetido nos demais lados do cubo a fim de resolvê-lo.

Passo 2: Obtenha uma face de uma mesma cor. Para tanto se deve inserir as 4 peças de vértice correspondente na camada onde foi feita a cruz.

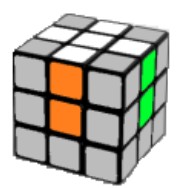

Figura 9: Posição do cubo ao final do Passo 1

Neste ponto teremos $1 / 3$ do cubo resolvido, como mostra a Figura 10

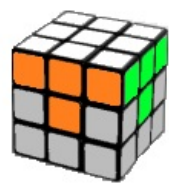

Figura 10: Posição do cubo ao final do Passo 2

Passo 3: Monte a camada intermediária. O objetivo aqui é inserir uma peça lateral da camada superior da camada intermediaria e não bagunçar o restante do cubo já resolvido. Ao fim deste passo teremos resolvido $2 / 3$ do cubo, como mostra a Figura 11 .

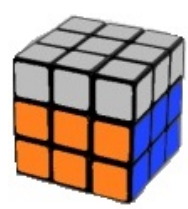

Figura 11: Posição do cubo ao final do Passo 3

Passo 4: Sem bagunçar o que já está resolvido, construir uma cruz na face oposta do cubo e colocar as peças laterais de cores correspondentes em suas posições corretas.

Passo 5: Neste momento o cubo deve se encontrar de forma equivalente a mostrada na Figura 12. Agora basta mover as últimas peças vértice desta camada para a posição correta e orientação correta. O cubo esta resolvido.

\section{Resolvendo do Cubo em Sala de Aula}

Ao se utilizar o cubo de Rubik em sala de aula é inevitável que se ensine a resolvê-lo. Utilizandose os movimentos e a estratégia da seção anterior, é possível ensinar de forma simples e com grande grau de eficácia. No entanto, seria mais interessante ensinar a resolvê-lo aos poucos, diluindo a solução do cubo ao longo de várias aulas à medida que se utiliza o cubo para ilustrar e discutir conteúdos de física como simetrias, leis de conservação, entropia e irreversibilidade. Dessa forma as atividades voltadas para a resolução do cubo não necessariamente precisam ocupar um tempo demasiadamente grande em sala de aula. Com isso em mente, abaixo se delineia uma proposta 


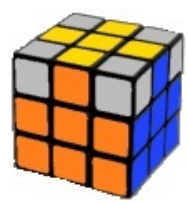

Figura 12: Posição do cubo ao final do Passo 4

de atividades que não devam ocupar mais que 20 minutos de cada aula.

Aula 1: Ensine os estudantes as 3 sequencias de movimento apresentadas na seção anterior.

Aula 2: Ensine os estudantes a usar os movimentos descritos para fazer uma cruz em um lado do cubo (Passo 1). Exercício: Completar o Passo 1 em cinco minutos.

A maioria dos estudantes deverá conseguir resolver este exercício com facilidade. Proponha aos estudantes que não consigam executar a tarefa, refazer o exercício no inicio da aula seguinte.

Aula 3: Ensine aos estudantes a realizar o Passo 2: fazer com que toda uma face do cubo tenha a mesma cor. Exercício: Completar o Passo 2 em cinco minutos.

A esta altura deverá ser claramente perceptível a natureza sequencial deste método de resolução do cubo, uma vez que os alunos não poderão executar o Passo 2 se não concluírem primeiro o Passo 1. Este é um ponto importante e deve ser bastante enfatizado, sendo que o docente pode fazer diversas referências a ele quando tratar de outros temas que são sequenciais por natureza. Essa rotina pode ser repetida sistematicamente para cada passo, observando-se o progresso dos alunos. Na aula seguinte após ensinar a executar o 5 passo, dê aos alunos cinco minutos para resolver todo o cubo de Rubik. A maioria dos estudantes deve conseguir faze-lo dentro deste prazo com pouco ou nenhuma das dificuldades que tenham apresentado semanas antes.

Para se demonstrar o papel da prática contínua, atividades com o cubo podem ser utilizadas regularmente desafiando os alunos a melhorar os seus melhores tempos de resolução.

\section{Benefícios do uso do cubo de Rubik no ensino}

Eis alguns benefícios que podem ser esperados no uso do cubo de Rubik em sala de aula:

Aumentar a autoconfiança, especialmente nos alunos com desempenho insuficiente. Em muitos casos pessoas que mostram proficiência na solução do cubo apresentam dificuldades na escola. Tais pessoas tendem a gostar da abordagem mais prática e demonstram disposição para gastar horas de seu tempo praticando e tentando melhorar. Incorporar este tipo de atividade no ensino de física pode estimular estes estudantes, mostrando que suas competências são importantes para o estudo da disciplina. Na outra ponta, os alunos que aprendem a resolver o cubo tendem a sentir-se bem consigo mesmas, uma vez que a maioria da população em geral não pode resolvê-lo. Pode se dizer que estes alunos sentem que se eles podem resolver o cubo, então certamente eles podem fazer qualquer outra coisa que os professores peçam a eles. Em outras palavras, eles aprendem que se trabalharem duro o suficiente, eles podem ser bem sucedido.

Promoção da aprendizagem cooperativa. Como tem sido observado no uso de jogos e atividades lúdicas no ensino os alunos geralmente preferem trabalhar com seus colegas de classe, desde que eles podem obter ajuda de qualidade (Ref. [9]). Os professores também descrevem bastante satisfação ao ver estudantes trabalharem juntos, como eles ajudam uns aos outros e como o desempenho destes estudantes aumenta (Ref. $[10,11,12])$.

Ilustrar métodos de resolução de problemas. $\mathrm{O}$ raciocínio sequencial necessário para resolver o cubo é aplicável a muitos outros tipos de problemas. Por exemplo, antes dos alunos poderem resolver para a densidade de um objeto, por exemplo, eles devem primeiro conhecer a sua massa e volume. Ao quebrar os problemas em etapas, mesmo os mais complexos pode ser resolvido (Ref. [4]). Na verdade, todo o progresso científico ocorre em passos incrementais, com uma descoberta levando a outra. Aprender a resolver o Cubo de Rubik é uma boa oportunidade para o professor discutir com seus alunos como o progresso científico e tecnológico ocorre.

Melhora a percepção espacial. O cubo de Rubik é uma excelente ferramenta para melhorar o raciocínio espacial, uma vez que para resolver o cubo não basta apenas memorizar uma solução, mas planejar a manipulação de objetos tridimensionais. Este raciocínio visuo-espacial é infelizmente muitas vezes negligenciado no ensino de ciências (Ref. [13]). Muitos problemas em física, matemática e engenharia são de natureza espacial. Da compreensão e raciocínio sobre os átomos em uma molécula (Ref. [14]), ao projeto de sistemas mecânicos e eletrônicos, tais como robôs, layout de um circuito integrado em microeletrônica, da distribuição e transmissão de tensões e forças de compressão em um sistema estrutural, continuamente se exige a capacidade de visualizar e raciocinar espacialmente.

Demonstra a necessidade de prática. Se os alunos resolveram o cubo uma vez e depois não forem convidados para resolvê-lo novamente, ao final de um ano ainda conseguiria fazê-lo? Muito provavelmente não. Na corrida para cobrir ementas extensas, é fácil ensinar algo uma vez e nunca mais voltar ao assunto. E se os alunos fazem não se lembrarem do conteúdo, então eles realmente 
não terão aprendido.

Ao praticar a resolução do cubo de Rubik, a necessidade da prática é reforçada . De muitas maneiras, o cubo fornece um modelo de como toda a aprendizagem deve progredir : Estudantes são apresentados com um problema aparentemente intransponível, em seguida, após trabalho duro, eles resolvem o problema após dividi-lo em etapas e seguem praticando para refinar esses passos. Somente através da prática contínua é alcançada a proficiência.

Fornece exemplo de aprendizagem verdadeira. Pode-se argumentar que a verdadeira aprendizagem ocorreu quando já não precisamos parar para pensar. Seja amarrar os sapatos, ou usar os talheres para comer, no dia a dia uma pessoa realiza um infinidade de atividades, sem realmente pensar em como fazê-las. Usualmente todas estas tarefas requerem atenção e trabalho para serem aprendidas. No entanto, após aprendidas e repetidamente executadas essas habilidades se tornam praticamente automáticas.

Eventualmente, os alunos tornam-se tão proficientes em resolver o cubo de Rubik que podem resolvê-lo sem realmente pensar sobre isso. Sua memória motora assume e eles resolvem o cubo sem praticamente sem raciocinar. Quando algo se torna automático ele é armazenado na memória de longo prazo (Ref. [15]), que é o objetivo de todo o aprendizado. Um dos principais objetivos da educação é ajudar os alunos a acumular conhecimento útil na memória de longo prazo e utilizar essa informação em ocasiões posteriores para resolver efetivamente os problemas.

\section{Conclusões}

Com suas 26 peças o cubo de Rubik apresenta algumas das propriedades mais essenciais da física contemporânea, tais como simetrias de padrões discretos, caracterizada por números quânticos; leis do movimento e não-comutatividade; teoremas de conservação e irreversibilidade. Por tais características, o cubo oferece oportunidades interessantes para ensinar conceitos físicos modernos, além de desenvolver habilidades de resolução de problemas. Além disso, há algo de especial sobre este Quebra-cabeça 3-D que parece cativar a imaginação mesmo dos estudantes mais apáticos, mostrando um enorme valor em atingir o potencial inexplorado de cada aluno.

\section{Referências}

[1] Jan J Elshout and Marcel VJ Veenman. Relation between intellectual ability and working method as predictors of learning. The Journal of Educational Research, 85(3):134-143, 1992.
[2] Daniel F Styer. Getting there is half the fun. American Journal of Physics, 66:105106, 1998.

[3] Patricia Heller and Mark Hollabaugh. Teaching problem solving through cooperative grouping. part 2: Designing problems and structuring groups. American Journal of Physics, 60(7):637-644, 1992.

[4] George Polya. A arte de resolver problemas. Rio de Janeiro: Interciência, 2, 1978.

[5] Hilda Borko, Margaret Eisenhart, Catherine A Brown, Robert G Underhill, Doug Jones, and Patricia C Agard. Learning to teach hard mathematics: Do novice teachers and their instructors give up too easily. Journal for research in mathematics education, 23(3):194-222, 1992.

[6] David Joyner. Adventures in group theory: rubik's cube, merlin's machine \& other mathematical toys. The Mathematical Intelligencer, 27(2):92-92, 2005.

[7] George Marx, Eva Gajzago, and Peter Gnadig. The universe of rubik's cube. European Journal of Physics, 3(1):39, 1982.

[8] Solomon W Golomb. Rubik's cube and a model of quark confinement. American Journal of Physics, 49(11):1030-1031, 1981.

[9] Marilia Gabriela de Menezes, Rejane Martins Novais Barbosa, and Zélia Maria Soares Jófili. Aprendizagem cooperativa: O que pensam os estudantes? Linguagens, Educação e Sociedade, 12(17):51-61, 2007.

[10] Maria Regina Gomes da Silva. Considerações sobre o trabalho em grupo na aula de matemática. Mimesis, 19(2):135-145, 1998.

[11] David JOHNSON, Roger JOHNSON, and Karl SMITH. A aprendizagem cooperativa retorna às faculdades. Change, Jul/Aug98, 30(4):p26, 2000.

[12] David W Johnson, Roger T Johnson, and Mary Beth Stanne. Cooperative learning methods: A meta-analysis, 2000.

[13] James H Mathewson. Visual-spatial thinking: An aspect of science overlooked by educators. Science Education, 83(1):33-54, 1999.

[14] Hsin-Kai Wu and Priti Shah. Exploring visuospatial thinking in chemistry learning. Science Education, 88(3):465-492, 2004.

[15] Timothy D Lee, Stephan P Swinnen, and Deborah J Serrien. Cognitive effort and motor learning. Quest, 46(3):328-344, 1994. 